europea académica y políticamente fuerte para la Salvaguarda de los Patrimonios Culturales y para la Investigación en Conservación-Restauración constituyendo el segundo tema inalienable de esta cultura binaria (nacional/continental).

¿Será la reformulación de las enseñanzas en esta materia que, sobre el terreno, hará viable el estatus de los conservadores-restauradores en el seno de las estructuras administrativas, o viceversa?

Una vez clarificado, nuevo, limpio y listo para romper este círculo vicioso: A lo largo de estas discusiones, es recurso muy frecuente aludir a los Documento de ECCO I al III (citados anteriormente) ya que alcanza un particular legítimo y es que este perfil, ya exhaustivo, ha sido elaborado por la misma clase profesional. La adhesión a este trabajo de los expertos conjuntamente con otros profesionales implicados con la Conservación-Restauración de Bienes Culturales, desde directores de Instituciones, a científicos pasando por historiadores de arte, representan hoy el primer compromiso colectivo de las "Desideratas" y compromisos consignados. La representatividad de los conservadores-restauradores en el seno de ECCO y el peso de su opinión en el seno de la Comisión Científica han sido considerablemente reforzada.

Se echa de menos que el perfil y las competencias del conservador-restaurador, en el seno del documento de Pavia, no estén explícitas en el texto y que remiten sistemáticamente al lector a los documentos de EC$\mathrm{CO}$ incluidos en los anexos.

Partiendo de un perfil de conservador-restaurador más que definido, los debates de estas Jornadas se pueden articular y se interrogan acerca de los roles que estos profesionales competentes deberán igualmente asumir en la sociedad, al servicio de la categoría de los Bienes Culturales de los cuales son especialistas.

A este respecto y en relación con las competencias sobre Prevención/Conservación/Restauración, otras facetas, lagunas hasta hoy, les han sido añadidas, como las de consultor/ creador/ejecutor en la concepción de un proyecto de conservación-restauración en todos los estadios de su organización, para satisfacer la integración real y la salvaguarda de las responsabilidades; como la de colaborador en la gestión cultural del Patrimonio, sobre todo en lo que concierne a las prioridades y puesta en marcha de las políticas nacionales racionales y "productivas" (no existe hoy día planificación más imponderable que la de la tutela y la comanditaria, que predican la eficacia y la rapidez de la ejecución pero no se interesa por cuestiones éticas ni por la fiabilidad de las intervenciones); como la de estratega de la comunicación, para asegurar el diálogo con el exterior y la sensibilización de los diferentes públicos y organismos de tutela a las cuestiones de Preservación; como la de pedagogo especializado en Conservación-restauración para paliar la ausencia resentida de formadores en esta materia; como la de gestor potencial de los sistemas educativos para reflexionar sobre la enseñanza y promoverla.

Lejos de ser una conquista de la dominación de la Conservación-Restauración, esta contribución del conservador-restaurador debería llegar por el contrario en el seno de un mejor medio interdisciplinar, considerando que una vez definida las calificaciones de este profesional, debería evitarse la superposición de cargos entre los compañeros de la Salvaguarda del Patrimonio al igual que las viejas querellas institucionales sobre las competencias mal administradas.

A la luz de los principios sintetizados en este resumen, se ha ratificado unánimemente el 21 de octubre de 1997 por la asamblea representativa de los expertos reunidos en $\mathrm{Pa}$ via (respondiendo de forma inesperada a los ambiciosos objetivos iniciales definidos por las Jornadas Europeas), la creación de una legislación apropiada y coherente a escala nacional o comunitaria; queda finalmente la puesta en marcha de estos acuerdos y de estas resoluciones, para que el tema a debate ancle en la realidad europea en la dimensión concreta, moral, social y política que revindica después de treinta años.

La verdadera inserción de la Conservaciónrestauración en el contexto administrativo no será una realidad hasta que las competencias de los conservadores-restauradores no sean asimiladas y dirigidas por los marcos reglamentarios de la Cultura, de la Educación, de la Finanzas y de la Justicia, de forma conjunta y coherente. En el contexto material e histórico de la Preservación de los Bienes Culturales, esta inserción no se producirá más que confrontando esta problemática en diferentes umbrales: los efectos de los cambios estrictamente mercantiles, el oportunismo y la política de prestigio, el artesana- do y la revalorización de las materias tradicionales, entre otras.

En la ausencia generalizada, a escala continental, de una política responsable y ajustada a la Conservación restauración, la reformulación y el reconocimiento del profesional competente que se ocupa directamente los Bienes Culturales puede, en efecto, contribuir a la defensa de un Patrimonio duradero y auténtico. Con la condición de la reglamentación de la actividad del conservadorrestaurador sea coordinada.

Pero no nos engañemos, con o sin el documento de Pavia, sí la Unión Europea no cuestiona su política económica con un trabajo de fondo sobre su identidad cultural, en pro de la protección, la interpretación y la divulgación de los patrimonios nacionales específicos, al igual que la valorización de los recursos humanos, seguirán siendo letras muertas.

\section{EL CONSERVADOR-RESTAURADOR DE BIENES CULTURALES: UNA PROFESIÓN A DEBATE}

Mª José González López

\section{Introducción}

Hoy día la profesión de restaurador de bienes culturales está en curso de transformación. Las desigualdades existentes entre los niveles formativos, el reconocimiento y las competencias de estos profesionales en los respectivos países de la Comunidad Europea, están ocasionando una toma de conciencia del problema existente, así como de la importancia que, para la salvaguarda del patrimonio cultural, adquiere esta figura profesional, tanto por parte de este colectivo, como por el de otras disciplinas implicadas, y también, por parte del mundo de la administración y político.

De hecho, las medidas que en las últimas décadas se están tomando al respecto nos indica cual es la nueva orientación y las exigencias que cada vez con más fuerza se demanda a este colectivo por parte de la sociedad.

Hoy día es impensable la concepción tradicional del restaurador que ejecuta su trabajo de forma aislada e intuitiva y con respuestas a todas las demandas que plantea el proceso de conocimiento del bien y su posterior tratamiento. 
Noticias y Comentarios

Esta concepción tradicional comienza a modificarse en la década de los ochenta con el documento presentado por el Grupo de trabajo para la formación en conservación y restauración del ICOM titulado: "El Conservador-Restaurador una definición de la profesión" publicado en el Congreso Trienal del ICOM celebrado en Copenhagen en 1984. Para posteriormente consolidarse con la Guía Profesional que elabora la Confederación Europea de Organizaciones de Conservadores-Restauradores (ECCO) en 1993/94.

Ambos documentos han sido considerados como textos básicos y punto de partida, conjuntamente con la Carta de Venecia de 1964, en las Jornadas Preservación del Patrimonio Cultural: hacia un perfil europeo del Conservador-Restaurador, celebradas en Pavia del 28 al 22 de octubre del presente ao, siendo por ello objeto de comentario y de síntesis ya que su contenido, facilitará la comprensión de las conclusiones alcanzadas por parte de los expertos en las Jornadas de Pavia.

\section{El conservador restaurador, una definición de la profesión}

La principal novedad que introduce este documento con respecto a la concepción tradicional de este profesional parte de la propia denominación de la profesión, ampliándose el concepto de restaurador de obras de arte, a conservador-restaurador de bienes culturales.

Esta distinción es claramente significativa y está estrechamente vinculada con las competencias y actividades que con mayor medida desempeñan y le son demandadas. Su campo de acción se ve ampliado desde el meramente cognoscitivo (examen/diagnosis) y operativo (aplicación de los tratamientos necesarios, conservación, restauración) a las derivadas de su preservación y salvaguarda (entorno, manipulación, mantenimiento) -conservación preventiva-.

El principal objetivo de este documento es la definición de la profesión y de sus competencias con vistas a instituir unos principios de partida que consientan a este colectivo establecer unos estatutos, al igual que ocurre con otras especialidades emparentadas (conservador, arqueólogo, arquitecto, etc), que le permitan el ejercicio de la profesión con la consideración y dignidad que merece, con independencia del ámbito donde se desem- peñe (museos, centros oficiales, empresas privadas, etc).

Desde el momento en que se toma conciencia por parte de la sociedad y también por parte de este colectivo, de la importancia que ejerce el restaurador en el Patrimonio Cultural de un país o región, desde el punto de vista que "opera" directamente sobre originales con un importante valor cultural, social, histórico, religioso y científico, y de consecuencia sobre el patrimonio a transmitir a generaciones venideras, se analiza el impacto que puede derivarse del desarrollo de su actividad ante :

- Responsabilidad ante la acción directa sobre los bienes patrimoniales con independencia de su valor o importancia históricoartística.

- Preservación de la integridad física del bien.

- Adecuación de la intervención, entendida como la aplicación de los tratamientos que requiere el bien, haciendo clara distinción entre los posible e imposible, trabajando para ello en estrecha colaboración con otros especialistas implicados.

- Reconocimiento del testimonio documental implícito del cual todo bien cultural es portador, así como su respeto y transmisión.

- Realización de un correcto examen metódico y científico que garantice, con su adecuada interpretación, la validez de las decisiones tomadas.

- Aplicación de una metodología científica (investigación de las fuentes, análisis, interpretación y síntesis) que avale la intervención y contribuya, desde el respeto de sus valores físicos y documentales, a su transmisión y comprensión.

- Implementación de la habilidad manual con el conocimiento teórico y con la capacidad de evaluar la situación a fin de adoptar las soluciones adecuadas.

- Interdisciplinariedad de la acción trabajando en equipo conjuntamente con otras disciplinas, estableciendo los mecanismos de diálogo adecuados para poder establecer las interpretaciones derivadas en un contexto exacto.

Está claro que las actividades que desempeña y se exige al conservador-restaurador en este documento, entran en conflicto las que desarrollan las profesiones artísticas y artesanales. La principal diferencia entre ambas, es que el conservador-restaurador no crea objetos culturales, sino que los conserva, restaura o mantiene, empleando para ello una metodología científico-técnica que parte de la investigación aplicada al conocimiento para la intervención.

Concluye este documento con una propuesta formativa que capacite al conservador-restaurador al correcto ejercicio de la profesión haciendo compatible la sensibilidad y capacitación manual, con el conocimiento de los materiales, de las técnicas y de la metodología científica aplicada. Siendo objetivo último, la formación de profesionales altamente cualificados capaces de abordar con metodología científica intervenciones complejas y lo que es también importante y novedoso, documentándolas con rigor a fin de que los datos derivados de este proceso formen parte de la vida de la obra contribuyendo a facilitar su comprensión y conocimiento, es decir a su difusión de forma racionalizada.

\section{Guía profesional: ECCO}

En 1993/94 la Confederación Europea de Organizaciones de conservadores-restauradores (ECCO) redacta un documento denominado Guía Profesional, que constituye hoy día un documento base y actualizado que define con rigor la ética y el perfil de competencias y formativo de esta figura profesional al que se acoge todo profesional asociado a esta Confederación. Este documento se estructura en tres grandes apartados: I. La Profesión, II. Código deontológico y III. Requisitos básicos para la educación en Conservación-Restauración,

Un análisis de este documento nos permite realizar una síntesis de su contenido.

En relación a la Profesión, el principal objetivo es la preservación del bien cultural (mueble). Para ello se establecen las siguientes competencias :

- Examen-diagnóstico: determinación de la composición y condición del bien cultural

- Conservación preventiva: Realización de acciones indirectas que permitan el uso y disfrute del bien en condiciones óptimas.

- Tratamientos de conservación: Ejecución de aquellas acciones directas encaminadas a retardar su deterioro. 
- Restauración: Realización de aquellas acciones directas que requiere el bien cultural para facilitar su compresión, siempre desde el respeto hacia su integridad estética, física e histórica.

Ampliándolas con las siguientes: Programas de inspección, asistencia técnica, dirección de proyectos, emisión de informes sin juicio sobre el valor comercial del bien, desarrollar programas educacionales en esta disciplina, difundir la información generada con su investigación o trabajo y por último, fomentar la comprensión de esta disciplina.

Es de interés destacar como este documento resalta la necesidad de elevar el nivel formativo de esta profesión, a un grado universitario o equivalente, para mantener su estatus profesional.

El Código Deontológico, al que se acoge todos los profesionales miembros de ECCO (art. I), establece 25 artículos estructurados en cuatro apartados: I. Principios generales para la aplicación del Código, II. Obligaciones hacía el bien cultural, III. Obligaciones de los propietarios y de los custodios legales y IV. Obligaciones hacia los Colegas y hacia la Profesión.

De su contenido, resaltaremos algunos de los aspectos más significativos relacionados con el ejercicio de esta profesión:

En relación al primer apartado: Principios generales para la aplicación del Código,

- Constituye una actividad de interés público y ha de ser practicada en consonancia con la legislación vigente (art. 2).

- Al trabajar sobre el bien cultural, es responsable directo ante su propietario y ante la sociedad, asistiéndole el derecho de desarrollar sin ningún obstáculo hacia su libertad e independencia (art. 3).

Del segundo de los apartados: Obligaciones hacía el bien cultural,

- Respeto absoluto hacia los valores estéticos, históricos y a la integridad física del bien cultural confiado a su cuidado (art. 5)

- Consideración hacia los requerimientos del uso social mientras preserva el bien cultural (art. 6).

- En su trabajo no debe influenciar el valor comercial del bien (art. 7).
- Deberá tener en consideración todos los aspectos de la conservación preventiva antes de abordar los trabajos físicos sobre el bien cultural, y limitará el tratamiento a lo estrictamente necesario (art. 8).

- Deberá emplear solamente productos, materiales y procesos que no perjudique al bien cultural, a su ambiente o a la gente, a fin de no interferir con ulteriores examen, tratamientos o análisis. Además, deberán ser compatibles con los materiales del bien cultural y ser tan fácil y completamente reversible como sea posible (art. 9).

- La documentación del bien cultural deberá incluir y dejar constancia del examen-diagnóstico, de la intervención y de cualquier otra información relevante (art. 10).

- Debe acometer únicamente aquel trabajo que le competa desarrollar; no debe empezar ni continuar un tratamiento si éste no es el mejor para el interés del bien cultural (art. II).

- Deberá enriquecer sus conocimientos y técnica con el proposito constante de perfeccionar la calidad de su trabajo profesional (art. 12).

- Para el mejor desarrollo de su trabajo deberá consultar con los especialistas adecuados, participando con ellos en el intercambio de información (art. 13).

- Proporcionar la asistencia posible en casos de emergencia (art. 14).

- No eliminará material de un bien cultural, a menos que sea indispensable para su preservación o interfiera sustancialmente con sus valores históricos y estéticos intrínsecos, debiendo documentar el proceso conservando el material eliminado (art. 15).

- Cuando el uso social sea incompatible con la preservación del bien cultural, discutirá con el propietario, o con los encargados de su custodia legal, si la realización de una reproducción del objeto puede ser una solución intermedia apropiada asesorándole al respecto (art. 16).

Del tercero de ellos: Obligaciones de los propietarios y de los responsables legales,

- Proporcionar información al propietario del bien sobre su cuidado y mantenimiento (art. 17).
- Está obligado al secreto profesional. Para hacer referencia a un bien cultural específico debe obtener el consentimiento del propietario o de las personas responsables de su custodia legal (art. 18).

Y por último del cuarto apartado: Obligaciones hacia los Colegas y hacia la Profesión,

- Mantener un espíritu de respeto hacia la integridad y dignidad de los colegas y de la profesión (art. 19).

- Participación, en la medida de sus posibilidades y de sus conocimiento, en la formación del personal a su cuidado, siendo responsable de los trabajos encomendados a su supervisión. (art. 20).

- Contribuir al desarrollo de la profesión mediante el intercambio de experiencias e información (art. 2I).

- Fomentar una mayor comprensión y un mayor conocimiento de la conservaciónrestauración (art. 22).

- Los datos relacionados con la conservación-restauración del bien son de su propiedad intelectual (sujeto a los términos de su contrato o empleo) (art. 23).

- No es compatible con su actividad la implicación con el comercio del bien cultural (art. 24).

- Con objeto de mantener la dignidad y credibilidad de la profesión deberá emplear únicamente las formas apropiadas de publicidad en relación con su trabajo (art. 25).

Sobre la Formación de estos especialistas, este documento plantea la necesidad de definir un programa formativo unánime en cuanto a filosofía, y contenido que abarque las distintas especialidades de esta disciplina y que incluya formación teórica y practicas aplicadas a casuísticas concretas, con objeto de que los profesionales estén suficientemente preparados y capacitados para desempear responsablemente el ejercicio de esta profesión de forma interdisciplinar con las profesiones afines y de desarrollar investigaciones aplicadas a la conservación-restauración y a las técnicas históricas.

Para ello plantea una formación a nivel universitario o equivalente a la que se acceda mediante un examen de admisión que valore la aptitud del candidato. Tras la conclusión 
Noticias y Comentarios

de estos estudios, que debería preferiblemente constar de cuatro aos, obtendría el título, previo examen final, en el que se hará constar la especialidad estudiada.

De igual forma, define los contenidos generales que debe abarcar la formación práctica y teórica:

- "La formación práctica debe incluir tratamientos sobre objetos originales particularmente idóneos a los fines instructivos. Los objetos elegidos deben proporcionar material para una buena documentación y ser caso de estudio incluyendo examen técni$\mathrm{co}$, diagnosis e informe de tratamiento.

Los estudios y prácticas de las técnicas históricas del arte y de los procesos de manufacturación de los materiales artísticos están encaminados a fomentar la mayor comprensión de los aspectos físicos, históricos y artísticos del bien cultural".

- Los contenidos teóricos deben plantearse desde un equilibrio entre las ciencias y las humanidades e incluir:

- Principios éticos fundamentales de la conservación/restauración.

- Ciencias (por ejemplo: química, física, biología, mineralogía, teoría del color).

- Humanidades (por ejemplo: historia, paleografía, historia del arte, arqueología, etnología, filosofía y estética).

- Historia de los materiales y de las técnicas artísticas incluyendo tecnología y procesos de manufacturación.

- Introducción a las causas de deterioro.

- Exposición y transporte del bien cultural.

- Teoría, métodos y técnicas de conservación y acciones preventivas.

- Teoría, métodos y técnicas de restauración.

- Introducción a los procesos de realización de reproducciones de objetos artísticos.

- Métodos de documentación de objetos artísticos incluyendo representación gráfica, procesadores de textos y técnicas fotográficas.

- Introducción a la investigación de trabajos científicos.
- Introducción a la historia y métodos de preservación de nuestro patrimonio cultural, y también, museología y conservación de monumentos y del entorno.

- Introducción a la legislación y administración.

\section{Conclusiones}

Creemos que las conclusiones adoptadas por los expertos participantes en las Jornadas celebradas en Pavia permiten poner en evidencia la problemática actual de la profesión sentando las bases para instar a los organismos e instituciones implicadas en las vías adecuadas de solución.

Si bien en la actualidad la diversidad de titulaciones y de nivel académico de estos profesionales, tanto entre los diferentes países comunitarios, como a nivel interno en un mismo país, está originando una serie de conflictos que dificultan la contratación, el intercambio de experiencias y la igualdad de estos profesionales de cara al desarrollo de su profesión en unas condiciones homogéneas, tanto a nivel interno de esta disciplina, como en paridad con las implicada en la salvaguarda del Patrimonio Histórico.

Ante esta circunstancia, hasta que se hagan realidad las conclusiones del documento de $\mathrm{Pa}$ via, queda por abordar el tema de la homologación de las titulaciones actualmente vigentes en los respectivos países de la Comunidad Europea. En este sentido consideramos importante, instar a las autoridades competentes a que valoren la viabilidad de homologar las actuales titulaciones vigentes de ConservaciónRestauración existentes en la Unión Europea (expedida por Centros Oficiales reconocidos con independencia del nivel académico obtenido), con objeto de que este profesional pueda ejercer su profesión en igualdad de condiciones y desde el respeto y consideración que merece su trabajo, como bien expresa las conclusiones de Pavia

\section{EL DOCUMENTO DE PAVÍA: EN BUSCA DE LA NORMALIZACIÓN}

José María Losada

Cuando comenzaron los trabajos que habrían de cristalizar en la reunión de Pavia, no es fácil que se tuviera la intuición de cuál iba a ser el resultado final de cinco días de intenso trabajo. Estamos seguros de que ni siquiera los más optimistas pudieron imaginar que se llegase tan lejos como se ha hecho, hasta el punto de alumbrar un documento que, seguramente, constituirá una referencia importante en el ámbito de la conservación de los bienes culturales.

El enunciado del encuentro ("...hacia un perfil europeo del restaurador de bienes culturales") invitaba a pensar que se orientase en un sentido que pudiera tener más de reivindicación profesional, de reconocimiento y defensa de un estatus específico. Pero el "Documento de Pavia" va mucho más allá en el desarrollo de una serie de puntos que se orientan hacia una normalización de la profesión del conservador-restaurador en el ámbito europeo, aunque los puntos 3 y 4 hagan referencia directa a esa definición de un perfil específico.

Este esfuerzo de normalización parece especialmente importante, por cuanto responde a cualquiera de las acepciones comunes del término, esto es, se refiere tanto al intento de "regularizar o poner en buen orden lo que no lo estaba", como a "tipificar o ajustar a un modelo o norma". Todo ello en relación con la formación o el ejercicio profesional, aspectos ambos que efectivamente no están bien regulados, en líneas generales, en ningún lugar de Europa. Precisan por tanto de una definición de normativa que permita una equiparación que, desde el punto de vista laboral, resultará absolutamente imprescindible en un espacio abierto común.

Entendida normalización como regularización, a ella se pueden adscribir los puntos I, 4, 5 y 6 del documento. El hecho de que en ellos se haga referencia, básicamente, a aspectos relacionados con la formación, no parece baladí ya que, sin una enseñanza cualitativamente irreprochable, es difícil que podamos disponer de profesionales capacitados para afrontar la delicada tarea de intervenir en los bienes que constituyen el patrimonio cultural. Se explicita además la necesidad de integrar equilibradamente teoría y práctica en el proceso formativo. Y todo ello partiendo de una premisa fundamental, la necesidad de reconocimiento de una disciplina específica, la conservación-restauración, como primer componente de la "puesta en orden" de las actuaciones sobre los bienes culturales.

Sin embargo, con ser estos aspectos del Documento singularmente importantes, quizá lo 